\title{
ExpoKids: An R-based tool for characterizing aggregate chemical exposure during childhood
}

\author{
Mona Dai $\mathbb{B}^{1,5} \cdot$ Susan Y. Euling ${ }^{2} \cdot$ Linda Phillips $^{3} \cdot$ Glenn E. Rice $^{4}$
}

Received: 9 January 2020 / Revised: 18 August 2020 / Accepted: 11 September 2020 / Published online: 5 October 2020

This is a U.S. government work and not under copyright protection in the U.S.; foreign copyright protection may apply 2020

\begin{abstract}
Background Aggregate exposure, the combined exposures to a single chemical from all pathways, is a critical children's health issue.

Objective The primary objective is to develop a tool to illustrate potential differences in aggregate exposure at various childhood lifestages and the adult lifestage.

Methods We developed ExpoKids (an R-based tool) using oral exposure estimates across lifestages generated by US EPA's Exposure Factors Interactive Resource for Scenarios Tool (ExpoFIRST).

Results ExpoKids is applied to illustrate aggregate oral exposure, for ten media, as average daily doses (ADD) and lifetime average daily doses (LADD) in five graphs organized across seven postnatal childhood lifestages and the adult lifestage. This data visualization tool conveys ExpoFIRST findings, from available exposure data, to highlight the relative contributions of media and lifestages to chemical exposure. To evaluate the effectiveness of ExpoKids, three chemical case examples (di[2-ethylhexyl] phthalate [DEHP], manganese, and endosulfan) were explored. Data available from the published literature and databases for each case example were used to explore research questions regarding media and lifestage contributions to aggregate exposure. Significance These illustrative case examples demonstrate ExpoKids' versatile application to explore a diverse set of children's health risk assessment and management questions by visually depicting specific media and lifestage contributions to aggregate exposure.
\end{abstract}

\section{Introduction}

Aggregate exposure assessments evaluate the combined exposures to a single chemical across multiple routes (oral,

Supplementary information The online version of this article (https:// doi.org/10.1038/s41370-020-00265-6) contains supplementary material, which is available to authorized users.

Susan Y. Euling

euling.susan@epa.gov

1 Oak Ridge Institute for Science and Education at US EPA, Office of Children's Health Protection (OCHP), Washington, DC, USA

2 US EPA, OCHP, Washington, DC, USA

3 US EPA, National Center for Environmental Assessment, Washington, DC, USA

4 US EPA, Center for Public Health and Environmental Assessment, Chemical \& Pollutant Assessment Division, Cincinnati, OH, USA

5 Present address: Harvard John A. Paulson School of Engineering and Applied Sciences, Cambridge, MA, USA dermal, inhalation) and multiple exposure media/pathways (food, drinking water, dust, etc.). Aggregate exposure assessments are often important in children's health risk assessments because children's unique behaviors and physiology can alter chemical exposure rates across different media, routes, and lifestages relative to the adult lifestage. Further, relative to adults, children exhibit increased susceptibility to some chemical exposures during development, defined as a series of temporally and spatially orchestrated events from zygote implantation to completion of puberty [1-3]. Generally, chemical intake rates may be higher per unit body weight for children than for adults [2].

Aggregate exposures are addressed differently among and within organizations. The Food Quality Protection Act (FQPA) requires the US Environmental Protection Agency (EPA) to "ensure... reasonable certainty that no harm will result to infants and children from aggregate exposure to the pesticide chemical residue" [4]. In response, EPA published guidance for evaluating pesticide aggregate exposures [5]. Similarly, amendments to the Toxic Substances Control Act require EPA to "describe whether aggregate or sentinel 
exposures to a chemical substance [are] considered" as part of risk assessment [6]. Other federal organizations have developed similar methods for assessing aggregate exposure [7]. Outside of the USA, aggregate exposure assessment approaches for evaluating pesticide and consumer product exposures have also been created [8-10]. While efforts to comprehensively consider aggregate exposures from all pathways and routes have been developed, implementation has been limited by data availability for individual chemicals.

We developed ExpoKids to visualize contributions of multiple oral media to aggregate exposures both within and across lifestages. To our knowledge, the development of such approaches has been limited. This tool can effectively visually communicate and compare aggregate exposure information using available exposure data. Although ExpoKids can be used independently, it was developed to work with the Exposure Factors Interactive Resource for Scenarios Tool (ExpoFIRST; https://cfpub.epa.gov/ncea/ efp/recordisplay.cfm?deid=344928). We first describe the development of ExpoKids. Next, we explore the use of ExpoKids to visually illustrate the contributions of ten different oral media to seven lifestage-specific aggregate exposure estimates displaying average daily dose (ADD) and lifetime average daily dose (LADD). ADD refers to the dose rate averaged over a specified exposure interval and expressed as a daily dose on a per unit body weight basis. LADD describes the dose rate averaged over an individual's anticipated lifetime. The effectiveness of ExpoKids is then evaluated by posing three routine exposure assessment questions that highlight the potential utility of ExpoKids for a variety of stakeholders interested in children's environmental exposures.

\section{Methods}

ExpoKids Version 1.0 was developed in R (Version 3.4.0) and can be used as an extension of ExpoFIRST to illustrate aggregate exposure estimates. ExpoFIRST is a standalone tool that utilizes the EPA's Exposure Factors Handbook (EFH): 2011 Edition to provide deterministic potential dose estimates for user-defined exposure scenarios [2]. The EFH summarizes available human exposure data, providing exposure factor estimates [2].

\section{ExpoFIRST Integration}

Using ExpoFIRST (Version 2.0), we estimated the ADDs from the intake of ten media (i.e., soil, dust, water, breastmilk, dairy, meat, fish, vegetables, fruit, and grains) [2]. ADD was chosen as the ExpoKids metric to capture typical exposures experienced by average Americans. ExpoFIRST
Table 1 ExpoKids recategorizes the Exposure Factors Handbook $(E F H)$ age groups into lifestages based on the EPA's Guidance on Selecting Age Groups for Monitoring and Assessing Childhood Exposures to Environmental Contaminants.

\begin{tabular}{llll}
\hline EFH age groups & $\begin{array}{l}\text { ExpoKids } \\
\text { lifestage }\end{array}$ & $\begin{array}{l}\text { Total years } \\
\text { in lifestage }\end{array}$ \\
\hline Childhood & $\begin{array}{l}\text { Birth to } \\
<1 \text { month }\end{array}$ & Young Infant $^{\text {a }}$ & 1 \\
1 to $<3$ months & & \\
3 to $<6$ months & & \\
6 to $<12$ months & & \\
1 to $<2$ years & Infant $^{\text {a }}$ & \\
2 to $<3$ years & & \\
3 to $<6$ years & Young child & 3 \\
6 to $<11$ years & Child & 5 \\
11 to $<16$ years & Young youth & 5 \\
16 to $<21$ years & Youth & 5 \\
21 to $<70$ years & Adult & 49 \\
Birth to & Lifetime & 70 \\
$<70$ years & & \\
\hline
\end{tabular}

"The "young infant" and "infant" lifestages are the only ExpoKids lifestages to combine multiple EFH/ExpoFIRST age groups.

itself did not evaluate aggregate exposure, but rather ran ADD estimates for each medium separately. Subsequently, we exported the medium-specific ADD estimates from ExpoFIRST into ExpoKids to develop aggregate exposure graphs. Central tendency oral ADDs ( $\mathrm{mg} / \mathrm{kg}$-day) for the EFH's ten children's age groups and the adult age group (Table 1) within the general population were estimated in ExpoFIRST for each medium using the following general equation [2]:

$$
\mathrm{ADD}=\frac{C \times \mathrm{IR} \times \mathrm{EF} \times \mathrm{ED}}{\mathrm{AT} \times \mathrm{BW}}
$$

Above, $C=$ concentration $(\mathrm{mg} / \mathrm{mL}$ or $\mathrm{mg} / \mathrm{g}), \mathrm{IR}=$ intake rate $(\mathrm{mL} / \mathrm{kg}$-day, $\mathrm{g} / \mathrm{kg}$-day, or $\mathrm{mg} / \mathrm{day}), \mathrm{EF}=$ exposure frequency (days/year), $\mathrm{ED}=$ exposure duration (years), $\mathrm{AT}=$ averaging time (days), and $\mathrm{BW}=$ body weight $(\mathrm{kg})$ [2]. Age-specific central tendency estimates (either mean or median, depending on the exposure factor) from the EFH were used for IR, EF, ED, AT, and BW [2]. Chemical concentrations $(C)$ were based on media-specific values from data identified in the scientific literature. ExpoFIRST allows users to define an unlimited number of potential scenarios for various populations and lifestages. In running ExpoFIRST for our illustrative case examples, we selected parameters representing general population exposure.

While we recognize that our assumptions have some limitations, they were selected to be consistent and compatible with ExpoFIRST outputs. For the case examples, we 
estimated average wet weight concentrations based on data representative of an entire medium, even if only a subset was sampled. For all food categories, we estimated exposure for "total" food groups (e.g., total fruits, total vegetables), developing "per capita" estimates for males and females combined. For drinking water, we estimated exposure to chemicals in community water sources, thereby assuming that contaminants were only present in tap water (i.e., not bottled water) to be consistent with general population exposure scenarios. Once ADDs were estimated from ExpoFIRST, chemical-specific tables were exported and reorganized into Excel tables displaying ADDs for all media by lifestage and then input into ExpoKids to create graphs (see user guide [S-1: Fig. S1, Tables S1-4] in Supplementary Information for details).

For children under 1 month of age, EFH values were unavailable for soil and dust ingestion; both intake rates were assumed to be $0 \mathrm{mg} /$ day. Furthermore, due to a lack of available data on formula-fed infants, only breastfeeding data for young infants was included in ExpoFIRST v2.0.

\section{ExpoKids development}

ExpoKids can create five unique graphical displays of ADD by lifestage, LADD by lifestage, percent ADD by lifestage, ADD for individual exposure pathways, and LADD for individual exposure pathways (Table 2). Since the EFH followed the EPA's Guidance on Selecting Age Groups for Monitoring and Assessing Childhood Exposures to Environmental Contaminants as available data allowed, ExpoKids followed a similar renaming structure for reorganizing age groups into lifestages [1]. The ingestion pathway, for instance, did not have data for all age groups under 1 year of age and the EFH collapsed these four age groups into one, named young infants in ExpoKids. The infant lifestage in ExpoKids treated the 1-3-year-old EFH age groups similarly. Thus, eleven ExpoFIRST/EFH age groups were converted to seven ExpoKids lifestages (Table 1). This facilitated comparisons among six childhood lifestages, as well as between childhood in total (birth to less than 21 years of age), adulthood (21 to less than 70 years of age), and lifetime (birth to less than 70 years of age) ADD. After the data tables were uploaded (R package: readxl), the melt function ( $\mathrm{R}$ package: reshape2) rearranged the data into a readable format for the statistical program to create stacked bar plots using the ggplot function (R package: tidyverse). The resulting eleven graphs (one all media graph and ten medium-specific graphs) displayed the estimated ADD values by lifestage. LADD values were then estimated by time-weighting each ADD value; i.e., each ADD was multiplied by a ratio of time spent within each lifestage divided by the total lifespan (70 years). LADDs were calculated in ExpoKids to streamline calculations and prevent the need for multiple data exportations into ExpoKids. Estimates were performed and verified by the first author (MD).

For each medium, ADD per lifestage of interest $\left(\mathrm{ADD}_{j}\right)$ was estimated from the age groups using the following equation:

$\mathrm{ADD}_{j}=\frac{\sum\left(\mathrm{ADD}_{i} \times Y_{i}\right)}{\sum Y_{i}}$

in which $\mathrm{ADD}_{i}$ is the ADD value from the ExpoFIRST age group within the relabeled lifestage of interest, $Y_{\mathrm{i}}$ is the length in years of that age group, $\Sigma Y_{\mathrm{i}}$ is the total number of years in the new lifestage, and i represents the age group within the lifestage being estimated.

LADD per lifestage $\left(\mathrm{LADD}_{j}\right)$ of interest for the media was estimated from:

$\operatorname{LADD}_{j}=\frac{\operatorname{ADD}_{j} \times Y_{j}}{\text { Lifespan }}$

ADDs were also converted to percentages to estimate the percent contribution of each medium within a lifestage using the following equation:

$\%$ Lifestage contribution ${ }_{j}=\frac{\mathrm{ADD}_{j}}{\sum \mathrm{ADD}_{j}} \times 100 \%$

\section{Case example data selection}

To evaluate the effectiveness of ExpoKids, we developed illustrative exposure scenarios involving environmental chemicals and explored three questions that often arise in exposure assessments:

(1) What are the relative contributions of specific media across lifestages to lifetime aggregate chemical exposure?

(2) Are there differences in exposures across lifestages for an essential nutrient that is associated with developmental toxicity at elevated exposure rates?

(3) How has an environmental regulation affected aggregate exposure rates to a chemical across lifestages?

Our criteria for selecting environmental chemicals to explore the above research questions included: (1) evidence of developmental toxicity after exposure; (2) evidence of at least one critical or sensitive window of exposure during postnatal development; and (3) available chemical-specific concentration data in at least four oral exposure media included in ExpoFIRST. Following general assessment procedures to identify appropriate data [11], we searched PubMed in September 2018 to identify peer reviewed articles with clear descriptions of their scientific methodology (see S-3: Table S1 for search terms). Next, we scanned the 
Table 2 Five lifestage and oral media data visualization graph types produced by ExpoKids: ADD by lifestage, LADD by lifestage, percent ADD by lifestage, ADD for an individual exposure pathway, and LADD for an individual exposure pathway.

Graph type Example graph

1. ADD by lifestage: compares lifestages and media as relative contributors to an individual's lifetime exposure.

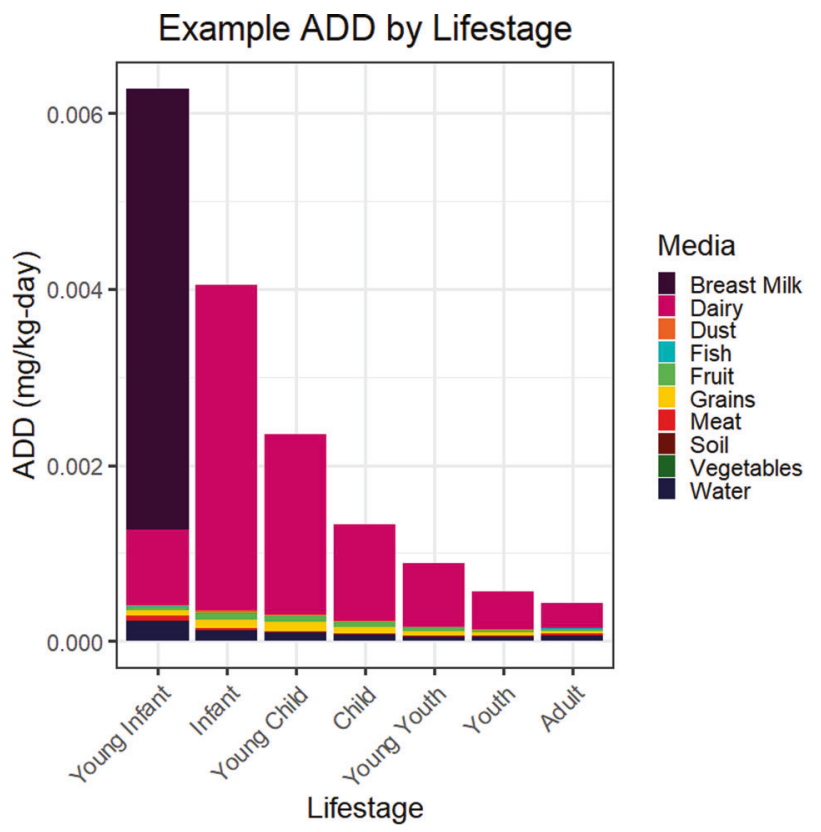

2. LADD by lifestage: compares lifestage and media ADD contributions scaled by the number of years an individual spends in each lifestage (time duration within a lifestage is considered).

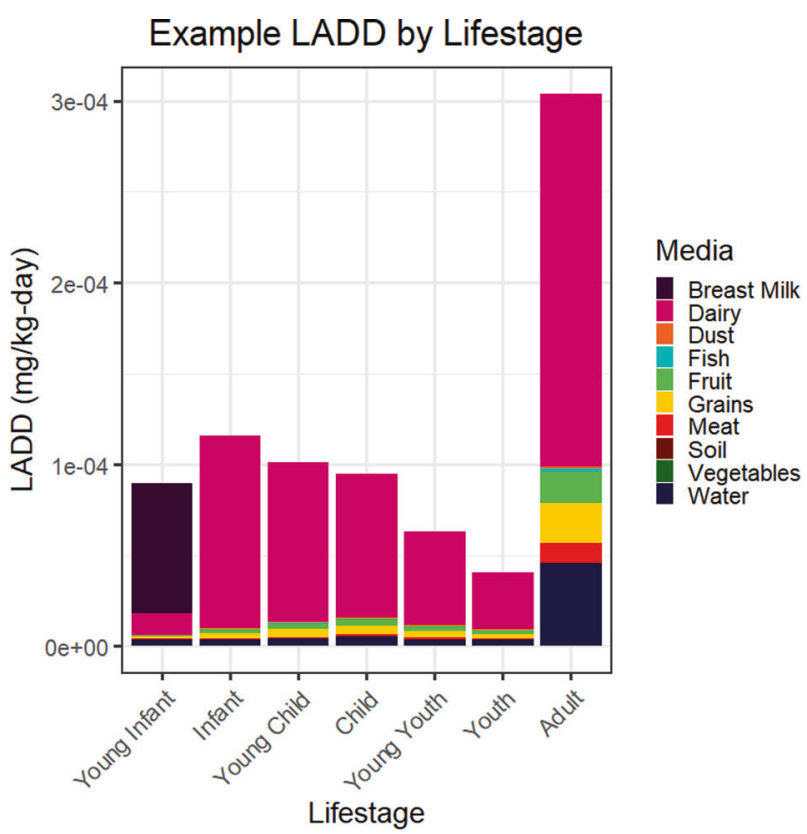

article titles and abstracts to ensure that the contents were useful for our illustrative case examples. We then applied the following inclusion criteria to the studies:

(1) Published between January 2000 and September 2018.

(2) Collected data in the USA, preferably nationwide.

(3) Quantitatively measured chemical levels directly in media.

(4) Conducted from a general population perspective.

All articles retrieved were reviewed to ensure that the general analysis, assumptions, and data were complete, and that variability was evaluated and characterized. To gauge the accuracy of the selected chemical concentrations, an 
Table 2 (continued)

Graph type

3. Percent ADD by lifestage: calculates relative ADD contributions within a lifestage as relative percentages within a lifestage.

4. ADD for an individual exposure pathway: focuses on illustrating the relative contribution between lifestages for a specific medium of interest (10 total).
Example graph
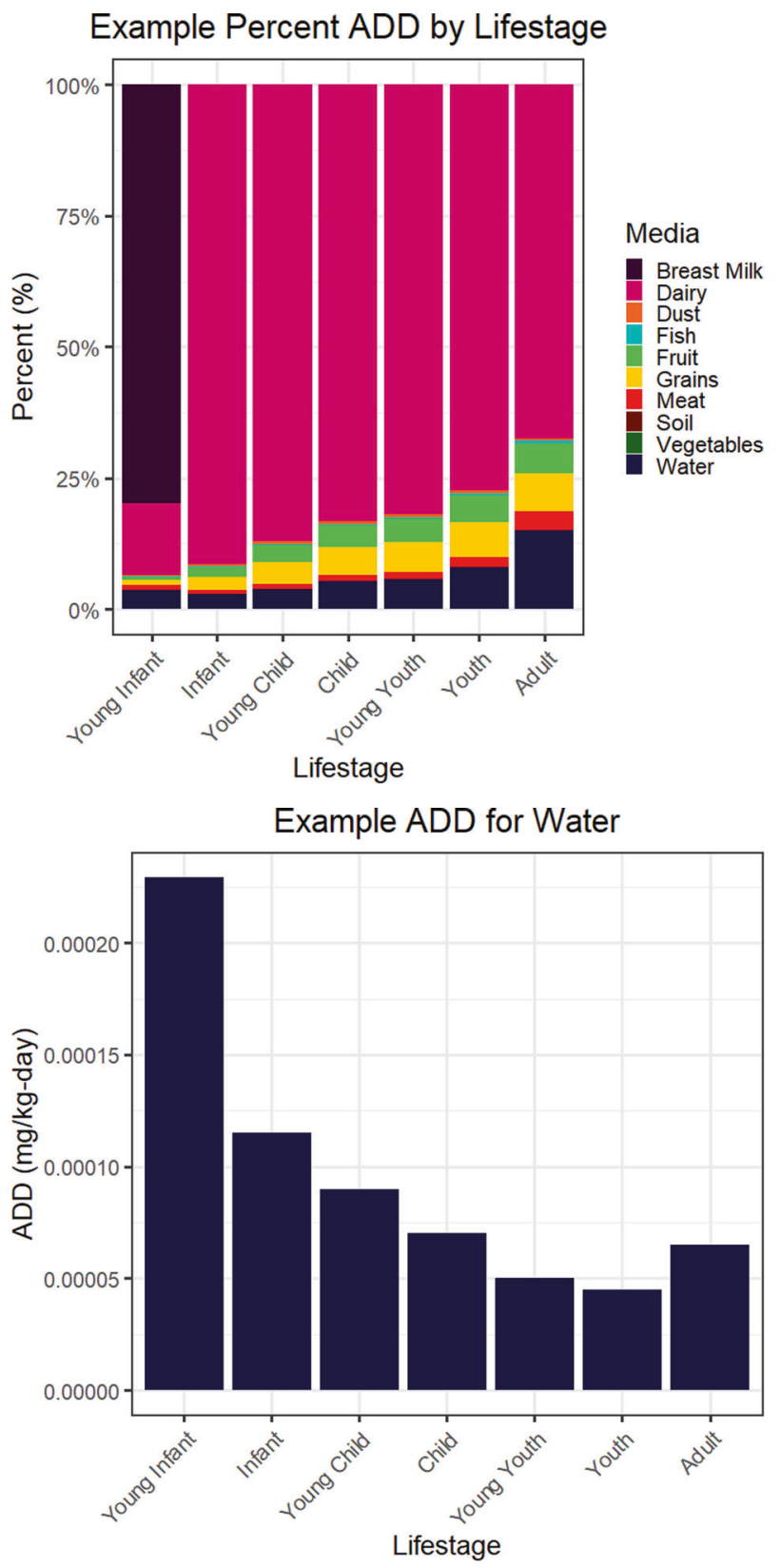

additional literature search was conducted on the largest relative ADD medium for each chemical (see S-3: Tables S2-4). Within each search, all relevant articles were collected for each chemical to compare their mean concentration inputs.

We next searched for candidate chemical concentration data. Our inclusion criteria included regularly maintained databases that used well-established methods with at least five years of monitored and/or measured US data. We also performed literature searches using keywords for the chemical of interest in each of the ten media to identify articles reporting media-specific concentrations. Mean media concentrations for chemicals were selected from a single data source keeping with the following 
Table 2 (continued)

5. LADD for an individual exposure pathway: focuses on illustrating the relative contribution between lifestages for a specific medium of interest (10 total) scaled by the number of years an individual spends in each lifestage.

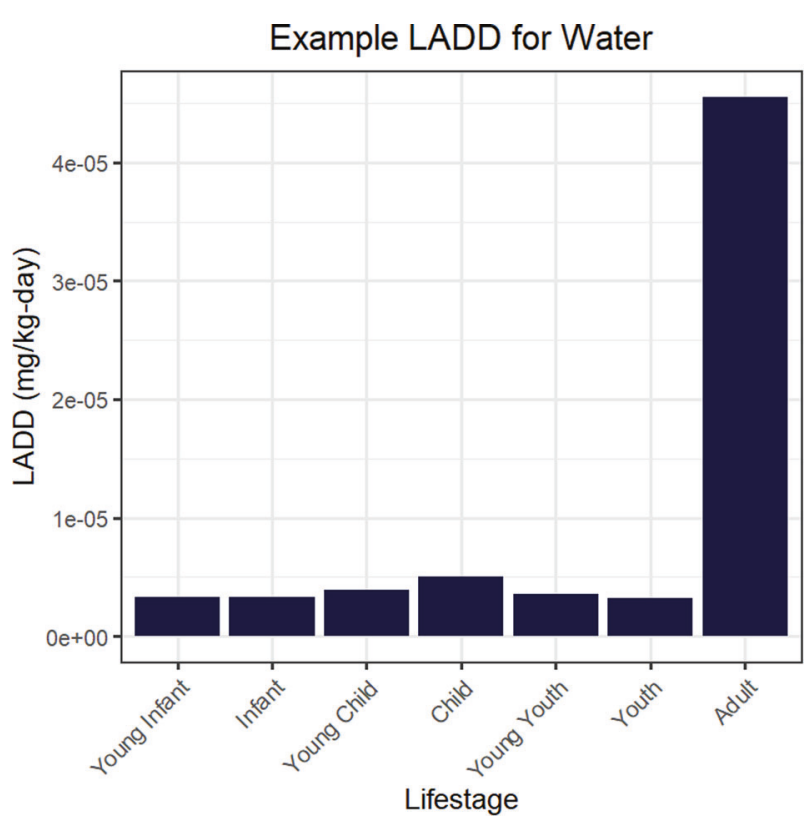

hierarchy-We preferred mean background concentration measures from recently published US nationwide databases and excluded measures from sampling locations with known elevated chemical concentrations (such as near chemical spills or industrial sites). Only measured chemical-specific concentrations were collected for each case example. Therefore, the accuracy of chemicalspecific concentrations relied heavily on measurement methods rather than models. Chemical-specific concentrations identified in the USA were prioritized over international chemical concentrations to avoid capturing differences in chemical production, use, or regulations from other countries. If an appropriate US database could not be found, we used the most recently published national study providing chemical concentrations measured in the media of interest. If nationwide data were not available, regional US studies were sought. When raw measurement data were collected, the arithmetic mean concentration was calculated for each medium. We also collected information regarding limit of detection (LOD), percent of samples above LOD, total number of samples measured $(n)$, median, and standard deviation (SD) (Tables 3-5). Since only one study or data source was chosen per medium, aggregate statistics were not calculated. Measurements below the LOD were excluded from mean estimates unless otherwise specified for the purposes of the illustrative case examples. Other non-detect approaches may be appropriate as well.

\section{Results}

\section{ExpoKids graphs}

ExpoKids visually conveys ADD and LADD findings to highlight the relative contributions of media and lifestages for environmental chemicals. ExpoKids' five aggregate exposure graph types illustrate differences across lifestages and media (Table 2). The aggregate ADD by lifestage graph illustrates aggregate media ingestion by lifestage to demonstrate the relative contribution of each medium within each lifestage. Likewise, the aggregate graph for LADD by lifestage depicts LADD contributions scaled by time spent in each lifestage. LADD graphs are useful for visualizing the relative average daily dose of each lifestage. For instance, although an aggregate ADD graph may illustrate higher exposure rates in younger childhood lifestages, the total years in childhood lifestages account for a shorter period of time compared to adulthood. As a result, time duration within a lifestage is considered when comparing estimated LADDs. The percent ADD graph displays ADD values on a cumulative percentage scale to compare relative media contributions within lifestages. The ADD and percent ADD graphs are also generated for childhood, adult, and lifetime exposures to facilitate comparisons. Lastly, these graphs are further individualized into ten ADD and LADD graphs per exposure pathway (20 total) that focus attention on specific media of interest. 
Table 3 Statistical descriptions of measured DEHP concentrations in media for the illustrative case example.

\begin{tabular}{|c|c|c|c|c|c|c|c|}
\hline Media & $n$ & Samples > LOD & LOD & Mean & Median & SD & Reference \\
\hline Dust $(\mathrm{mg} / \mathrm{g})$ & 11 & $100 \%$ & $0.14-278 \mathrm{ng} / \mathrm{mL}$ [dust/hexane: acetone] & $9.73 \mathrm{E}-02$ & $7.31 \mathrm{E}-02$ & $1.11 \mathrm{E}-01$ & Subedi et al. [16] \\
\hline Soil (mg/g) & 6 & $100 \%$ & $0.2 \mu \mathrm{g} / \mathrm{mL} 1$-butanol buffer & $9.39 \mathrm{E}-03$ & $8.63 \mathrm{E}-03$ & $2.19 \mathrm{E}-03$ & Lin et al. [17] \\
\hline Water $(\mathrm{mg} / \mathrm{mL})$ & 15 & $13 \%$ & $1.76 \mathrm{E}-06$ & $2.56 \mathrm{E}-06$ & N/A & N/A & Loraine et al. [18] \\
\hline Breastmilk (mg/mL) & 21 & $100 \%$ & $\begin{array}{l}\mathrm{MEHP}=6.18 \mathrm{E}-7 \\
\mathrm{MEOHP}=1.03 \mathrm{E}-7 \\
\mathrm{MEHHP}=1.03 \mathrm{E}-7\end{array}$ & $7.07 \mathrm{E}-04$ & $1.91 \mathrm{E}-04$ & $8.87 \mathrm{E}-04$ & Hartle et al. [19] \\
\hline Dairy (mg/g) & 11 & $100 \%$ & $3.70 \mathrm{E}-06$ & $1.26 \mathrm{E}-04$ & $6.97 \mathrm{E}-05$ & $1.25 \mathrm{E}-04$ & Schecter et al. [20] \\
\hline Meat (mg/g) & 13 & $69 \%$ & $3.70 \mathrm{E}-06$ & $1.01 \mathrm{E}-04$ & $7.00 \mathrm{E}-06$ & $3.18 \mathrm{E}-04$ & Schecter et al. [20] \\
\hline Fish $(\mathrm{mg} / \mathrm{g})$ & 5 & $80 \%$ & $3.70 \mathrm{E}-06$ & $3.14 \mathrm{E}-05$ & $3.96 \mathrm{E}-05$ & $2.60 \mathrm{E}-05$ & Schecter et al. [20] \\
\hline Vegetables (mg/g) & 5 & $40 \%$ & $3.70 \mathrm{E}-06$ & $5.09 \mathrm{E}-06$ & $1.85 \mathrm{E}-06$ & $8.70 \mathrm{E}-06$ & Schecter et al. [20] \\
\hline Fruit $(\mathrm{mg} / \mathrm{g})$ & 5 & $40 \%$ & $3.70 \mathrm{E}-06$ & $5.09 \mathrm{E}-06$ & $1.85 \mathrm{E}-06$ & $8.70 \mathrm{E}-06$ & Schecter et al. [20] \\
\hline Grains (mg/g) & 7 & $100 \%$ & $3.70 \mathrm{E}-06$ & $6.16 \mathrm{E}-05$ & $5.06 \mathrm{E}-05$ & $4.25 \mathrm{E}-05$ & Schecter et al. [20] \\
\hline
\end{tabular}

Table 4 Statistical descriptions of measured manganese concentrations in media for the illustrative case example.

\begin{tabular}{|c|c|c|c|c|c|c|c|}
\hline Media & $n$ & Samples > LOD & LOD & Mean & Median & SD & Reference \\
\hline Dust (mg/g) & 661 & N/A & N/A & $2.22 \mathrm{E}-01$ & $1.60 \mathrm{E}-01$ & $2.45 \mathrm{E}-01$ & Gulson et al. [31] \\
\hline Soil (mg/g) & 329 & N/A & N/A & $3.41 \mathrm{E}-01$ & $1.80 \mathrm{E}-01$ & $1.90 \mathrm{E}-01$ & Gulson et al. [31] \\
\hline Water $(\mathrm{mg} / \mathrm{mL})$ & 416 & $57 \%$ & $9.00 \mathrm{E}-07$ & $6.35 \mathrm{E}-05$ & $2.80 \mathrm{E}-06$ & $2.19 \mathrm{E}-04$ & Lindsey et al. [33] \\
\hline Breastmilk (mg/mL) & 20 & N/A & N/A & $2.71 \mathrm{E}-06$ & N/A & $1.12 \mathrm{E}-06$ & Klein et al. [32] \\
\hline Dairy (mg/g) & 759 & $25 \%$ & $3.0 \mathrm{E}-4$ to $4.0 \mathrm{E}-4$ & $8.19 \mathrm{E}-04$ & $1.30 \mathrm{E}-04$ & $1.46 \mathrm{E}-03$ & TDS 2006-2013 [34] \\
\hline Meat $(\mathrm{mg} / \mathrm{g})$ & 1725 & $65 \%$ & $3.0 \mathrm{E}-4$ to $4.0 \mathrm{E}-4$ & $1.66 \mathrm{E}-03$ & $1.70 \mathrm{E}-03$ & $1.06 \mathrm{E}-03$ & TDS 2006-2013 [34] \\
\hline Fish (mg/g) & 224 & $46 \%$ & $3.0 \mathrm{E}-03$ & $9.63 \mathrm{E}-04$ & $4.20 \mathrm{E}-04$ & $1.10 \mathrm{E}-03$ & TDS 2006-2013 [34] \\
\hline Vegetables (mg/g) & 1631 & $99 \%$ & $2.0 \mathrm{E}-4$ to $4.0 \mathrm{E}-4$ & $1.79 \mathrm{E}-03$ & $1.20 \mathrm{E}-03$ & $1.31 \mathrm{E}-03$ & TDS 2006-2013 [34] \\
\hline Fruit $(\mathrm{mg} / \mathrm{g})$ & 1055 & $79 \%$ & $2.0 \mathrm{E}-4$ to $4.0 \mathrm{E}-4$ & $1.62 \mathrm{E}-03$ & $4.45 \mathrm{E}-04$ & $3.45 \mathrm{E}-03$ & TDS 2006-2013 [34] \\
\hline Grains (mg/g) & 1484 & $99 \%$ & $3.0 \mathrm{E}-4$ to $4.0 \mathrm{E}-4$ & $7.34 \mathrm{E}-03$ & $4.90 \mathrm{E}-03$ & $7.14 \mathrm{E}-03$ & TDS 2006-2013 [34] \\
\hline
\end{tabular}

\section{Evaluating the effectiveness of ExpoKids}

Exposure assessment questions highlight the breadth of possibilities that may be examined by ExpoKids and emphasize the importance of aggregate exposure assessments when evaluating children's health. The results presented in the following case examples are for illustrative purposes only; alternate concentration estimates may produce different results. The estimated ADD values can be found in S-3: Tables S5-7.

\section{Question 1: What are the relative contributions of specific media across lifestages to lifetime aggregate chemical exposure?}

Di[2-ethylhexyl] phthalate (DEHP) was selected to illustrate this first question because of the chemical's developmental toxicity and historic uses [12]. DEHP, a "developmentally toxic phthalate", is associated with adverse reproductive effects following exposures during development [13]. DEHP assessments for various exposure sources (i.e., food, water, and commercial products) have been conducted by multiple groups [12-15]. Based on rat studies in which male reproductive effects were observed following gestational exposures, critical windows of exposure for phthalates and male reproductive developmental effects have been identified ranging from the most sensitive gestational exposure window, the moderately sensitive postnatal window, and the least sensitive adulthood window [13].

DEHP concentrations were extracted from five studies for the ten media of interest (Table 3). Five individual PubMed searches relating phthalates and the respective media of interest were conducted, retrieving 650 total articles. All extracted values were from regional US studies, except for soil concentration. The average DEHP concentration in dust was estimated based on phthalate dust samples collected in eleven homes from five geographically diverse US states in 2016 [16]. Since US DEHP soil data could not be identified, data collected from three fields in China over an unspecified time period were used [17]. Loraine et al. [18] reported DEHP concentrations in finished (treated) drinking water samples collected from California water treatment plants from 2001 to 2002. Hartle et al. [19] 
Table 5 Statistical descriptions of measured endosulfan sulfate concentrations (positive detects only) for the illustrative case example from USDA's Pesticide Database Program (PDP) [44].

\begin{tabular}{|c|c|c|c|c|c|c|}
\hline Media & $n$ & Samples $>$ LOD & LOD & Mean & Median & SD \\
\hline \multicolumn{7}{|c|}{ Pre Phase-Out: 1994-2010 } \\
\hline Dairy (mg/g) & 5220 & $14.80 \%$ & $3.00 \mathrm{E}-08$ to $1.00 \mathrm{E}-06$ & $2.55 \mathrm{E}-06$ & $2.00 \mathrm{E}-06$ & $7.45 \mathrm{E}-03$ \\
\hline Meat $(\mathrm{mg} / \mathrm{g})$ & 1176 & $1.80 \%$ & $3.00 \mathrm{E}-07$ to $2.00 \mathrm{E}-06$ & $1.48 \mathrm{E}-05$ & $4.80 \mathrm{E}-06$ & $2.67 \mathrm{E}-02$ \\
\hline Fish $(\mathrm{mg} / \mathrm{g})$ & 1479 & $6.60 \%$ & $1.00 \mathrm{E}-06$ & $5.26 \mathrm{E}-06$ & $2.70 \mathrm{E}-06$ & $6.03 \mathrm{E}-03$ \\
\hline Vegetables (mg/g) & 61805 & $11.70 \%$ & $2.00 \mathrm{E}-06$ to $4 \mathrm{E}-04$ & $3.80 \mathrm{E}-05$ & $2.00 \mathrm{E}-05$ & $7.49 \mathrm{E}-02$ \\
\hline Fruit $(\mathrm{mg} / \mathrm{g})$ & 39871 & $4.60 \%$ & $1.00 \mathrm{E}-06$ to $6.80 \mathrm{E}-02$ & $2.16 \mathrm{E}-05$ & $1.20 \mathrm{E}-05$ & $2.63 \mathrm{E}-02$ \\
\hline Grains (mg/g) & 558 & $0.20 \%$ & $1.00 \mathrm{E}-06$ & $2.00 \mathrm{E}-06$ & $2.00 \mathrm{E}-06$ & $0.00 \mathrm{E}+00$ \\
\hline \multicolumn{7}{|c|}{ Post Phase-Out: 2011-2016 } \\
\hline Vegetables (mg/g) & 21355 & $4.40 \%$ & $2.00 \mathrm{E}-06$ to $4.00 \mathrm{E}-04$ & $2.53 \mathrm{E}-05$ & $1.40 \mathrm{E}-05$ & $3.49 \mathrm{E}-05$ \\
\hline Fruit $(\mathrm{mg} / \mathrm{g})$ & 7415 & $1.50 \%$ & $1.00 \mathrm{E}-06$ to $6.80 \mathrm{E}-02$ & $2.26 \mathrm{E}-05$ & $1.90 \mathrm{E}-05$ & $1.28 \mathrm{E}-05$ \\
\hline
\end{tabular}

Fig. 1 DEHP illustrative case example graphs: ADD by lifestage graphs are shown in the top row and cumulative percentage ADD by lifestage graphs are shown in the bottom row.

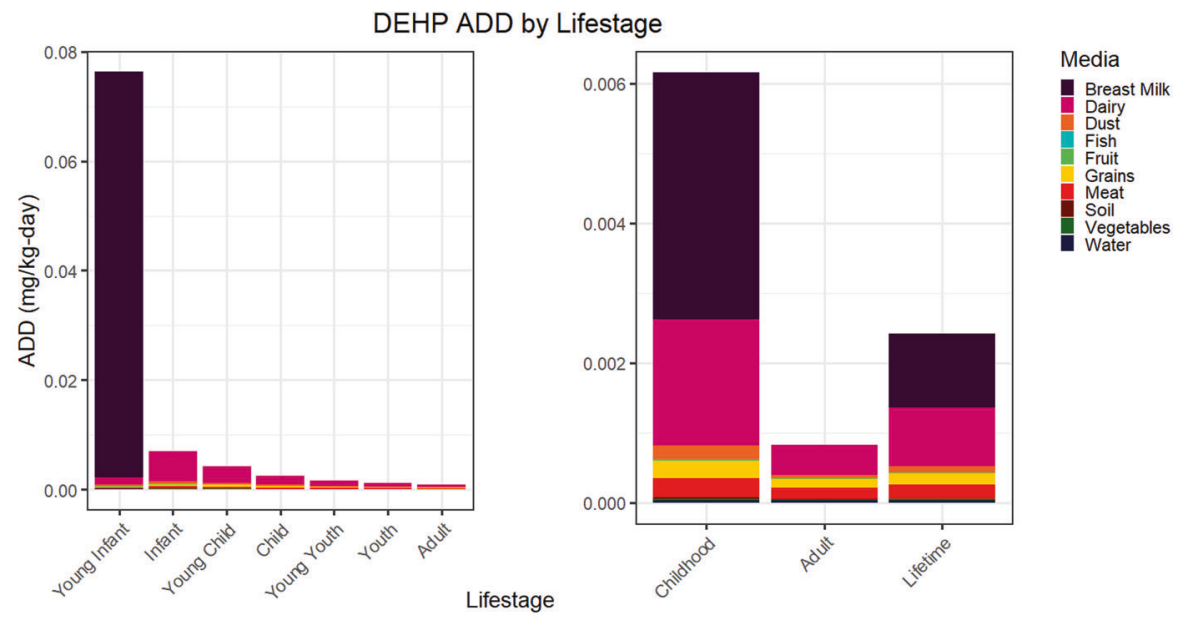

DEHP Percent ADD by Lifestage
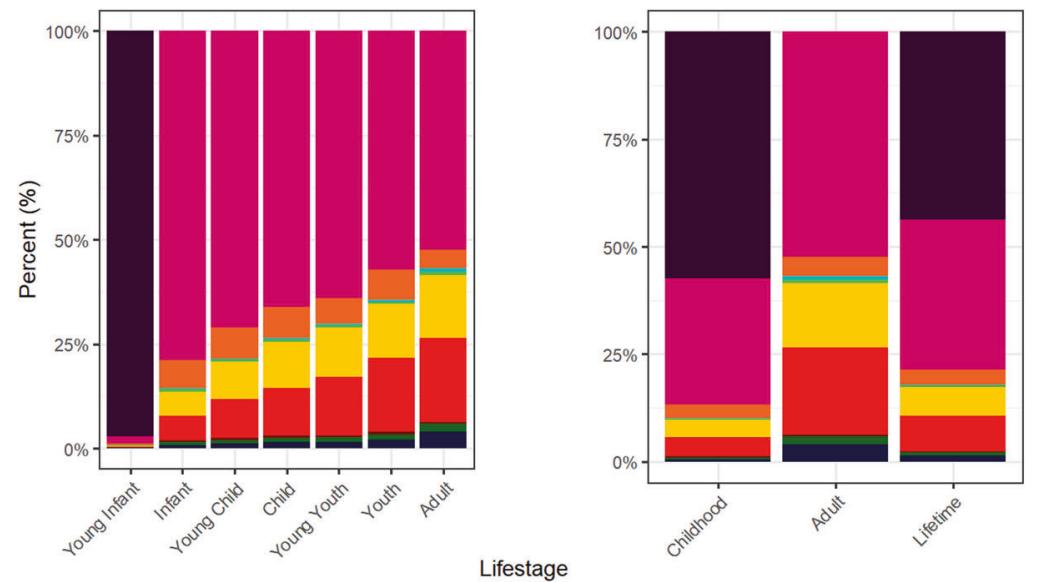

analyzed 21 human milk samples from 2015 in California; samples below the LOD were input as LOD $/ \sqrt{2}$. Unlike the other studies, three DEHP metabolites (MEHP, MEOHP, and MEHHP) were measured in breastmilk as a proxy for DEHP [19]. DEHP concentration in breastmilk was estimated by summing these average metabolite levels [19]. All food (dairy, meat, fish, vegetables, fruit, and grains) concentrations were extracted from Schecter et al. [20]. While relying on one study for all food groups provided consistency in data collection methodology, Schecter 
et al. sampled few food items overall $(n=41)$ from one state (New York) and fruits and vegetables were classified together [20].

The DEHP ExpoKids graphs (Fig. 1) show breastmilk consumption to be the dominant DEHP exposure medium in this illustrative case example based on an intake rate per kilogram body weight basis; among the included media, over $90 \%$ of the predicted DEHP exposure was from breastmilk intake for young infants. Young infants had the highest exposure rates to DEHP among all lifestages in this case study. After this lifestage, infants had the next highest aggregate ADD estimate, with declines in ADD in subsequent lifestages. Among all media evaluated, dairy intake accounted for over 50\% of estimated DEHP exposures across all lifestages except for young infants. In general, the relative contribution of all media within each lifestage remained relatively constant for individuals older than 1 year of age. Overall, during childhood, both breastmilk and dairy contributed more to ADD than other media evaluated.

\section{Question 2: Are there differences in exposures across lifestages for an essential nutrient that is associated with developmental toxicity at elevated exposure rates?}

Manganese was chosen as an illustrative case example for this question. The National Academies of Sciences (NAS) set a specified range for manganese between the lower limit or age-dependent adequate intake (AI) value and the tolerable upper limit (UL) value for discrete age groups to address low dose nutritional benefits and high dose neurotoxicity [21, 22]. Food is a major source of manganese intake for humans; as a result, assessing average dietary intake is important [22]. Laboratory animal studies with manganese oral doses during either gestational only, postnatal only, or gestational and postnatal developmental exposure windows reported developmental (e.g., skeletal development and growth) and neurodevelopmental toxicities [23-25]. These studies indicated that both the perinatal and the postnatal periods are critical windows of exposure. Epidemiologic studies also reported an increase in neurodevelopmental effects associated with increased prenatal or early postnatal manganese exposure [26, 27]. The EPA's Integrated Risk Information System (IRIS) RfD for manganese is $0.14 \mathrm{mg} / \mathrm{kg}$-day, based on central nervous system effects and is consistent with the UL value reported by NAS based on its review of the dietary literature $[21,22]$. Other agencies have also conducted health assessments of orally ingested manganese [28-30].

Table 4 lists average manganese concentration inputs to ExpoFIRST. Two studies on dust, soil, and breastmilk were identified from two PubMed searches that returned
464 articles. Since no US studies were available, dust and soil manganese concentration values were extracted from Gulson et al. [31] who sampled dust and soil collected from 108 households in Australia between 2001 and 2006. Mean manganese concentrations in breastmilk were estimated by Klein et al. [32] based on twenty 2013 breastmilk samples collected in Massachusetts. Mean manganese concentrations from 416 water samples from the United States Geological Survey's (USGS) National Water Quality Assessment's (NAWQA) dataset from 2013 to 2014 were also identified [33]. Since NAWQA reported median instead of mean manganese concentrations, these estimates were less outlier influenced [33]. USGS assigned half of the non-detect concentrations to be $0.05 \mathrm{mg} / \mathrm{L}$ (the LOD value) and the other half to be $0.025 \mathrm{mg} / \mathrm{L}$ (half of the LOD value) [33]. While this method may have overestimated manganese concentrations in water if the true concentrations were zero, this was unlikely since manganese naturally occurs in the environment [21]. The most recent FDA Total Diet Study (TDS) data collected from 2006 to 2013 were downloaded for food name, number of detects, LOD, and other summary statistics [34]. Each food commodity was sorted into its appropriate category based on its FDA categorization in the TDS food/analyte matrix before the mean manganese concentration for each food group was estimated [34]. All food commodities were assigned to a food category and average concentrations within each medium were estimated.

Figure 2 presents aggregate ADD manganese ExpoKids graphs based on inputs from Table 4. Manganese intake rates for all lifestages were between the AI and the UL, except for adults whose estimated rates were slightly below the AI level. Across lifestages, ADD values generally declined with age. Among the reported media, almost every lifestage followed a similar media ADD contribution pattern. Based on available data, oral ingestion from non-food sources were relatively low overall. However, the estimate for water ingestion may have been underestimated for young infants since ExpoFIRST v2.0 only included data on breastfeeding. In fact, previous studies reported that manganese intake rates from water in formula-fed young infants yielded the highest calculated dose compared to other lifestages [35]. This limitation of ExpoFIRST v2.0 may have led to underestimated manganese exposure rates in this lifestage. Instead, this illustrative case example predicted grains as the largest contributor to ADD across all lifestages. Consequently, the second row in Fig. 2 focuses specifically on ADD and LADD for grains, illustrating that ADD values are highest among infants and decline among subsequently older lifestages. Moreover, the contributions to manganese during adulthood to LADD were larger than other lifestage LADDs due to the longer duration of the adult lifestage. 
Fig. 2 Manganese illustrative case example graphs: ADD by lifestage graphs are shown in the top row and LADD by lifestage for exposure to grains is shown in the bottom row.
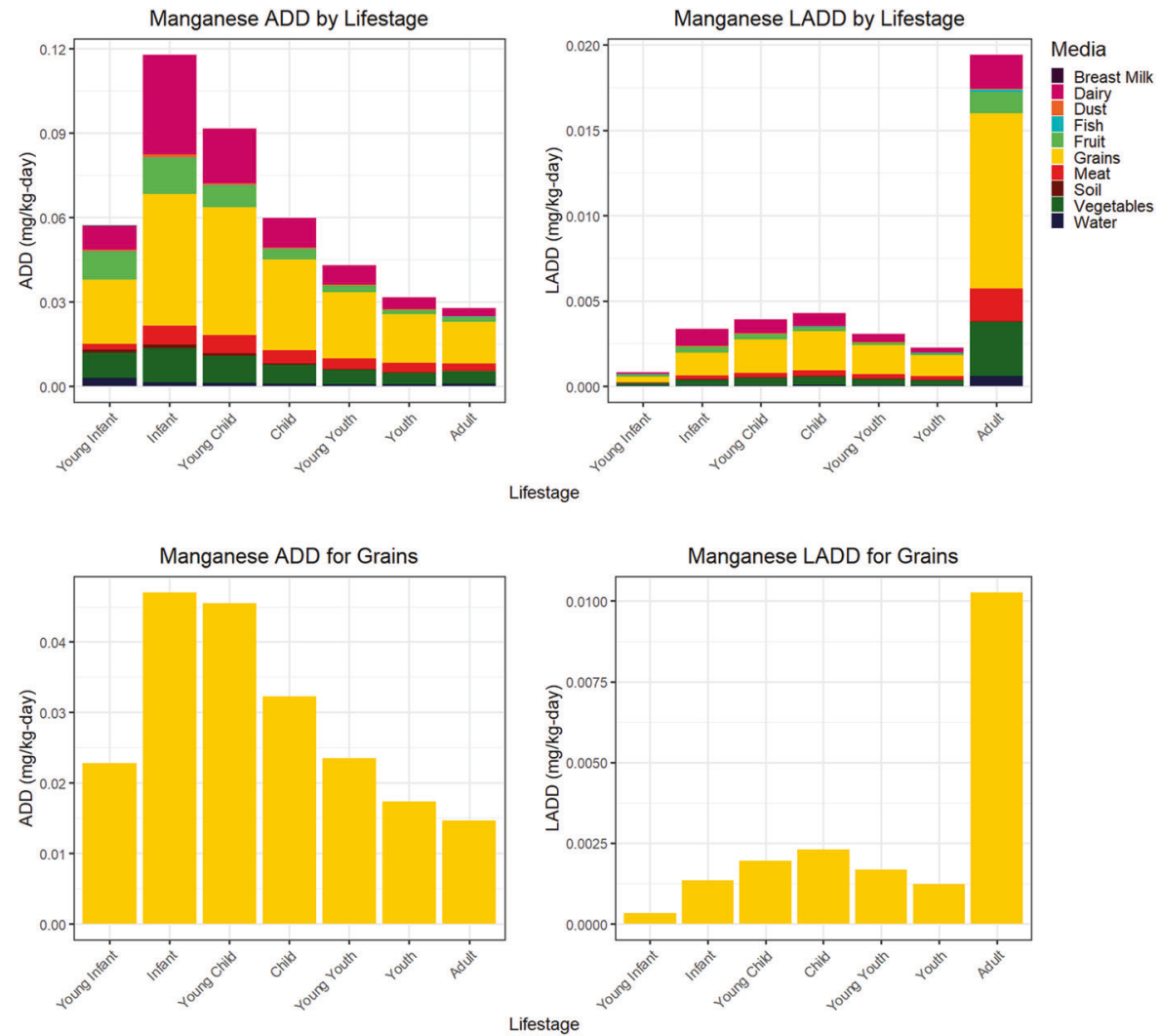

\section{Question 3: How has an environmental regulation affected aggregate exposure rates to a chemical across lifestages?}

Endosulfan, an organochlorine insecticide, was selected to ask whether a policy change, in this case a US federal intervention, affected lifestage or media-specific aggregate oral exposure rates using ExpoKids. Endosulfan was commercially applied on crops starting in the 1950s [36]. US EPA assessed the endocrine disrupting chemical's human health risks in 2002 and revised its human health and ecological risk assessments in 2007 [36]. Health assessments by international agencies have investigated the effects of endosulfan intake in food and water [37-39]. Exposure to endosulfan during prenatal and postnatal development can lead to neurodevelopmental effects [40]. Furthermore, high levels of endosulfan in the cord blood and breastmilk of pregnant women have been reported [41, 42]. Although data gaps exist in the understanding of specific critical windows of exposure, an animal study identified gestational and lactational exposure as sensitive developmental windows for endosulfan, leading to disruption of the nigrostriatal dopamine system development in male offspring [43]. As a result, historic endosulfan occurrence data can help explore the impacts of a regulatory decision that banned all uses of this pesticide in 2016 following a formal phase-out agreement in 2010 [36].
Dietary exposure to endosulfan before and after the 2010 phase-out was compared using data from the US Department of Agriculture's (USDA) Pesticide Data Program (PDP) [44]. Specifically, trace detections of endosulfan sulfate (the primary metabolite of endosulfan degradation in soil and sediments) in food commodities were evaluated from PDP reports downloaded for all available years (1994-2016) [45]. No data outside of this timeframe were available at the time of data collection. Table 5 shows concentration values (positive detects only) for ExpoKids media sources tested for endosulfan sulfate residue for the years 1994-2010 and 2011-2016. PDP measurements were extracted to estimate mean endosulfan sulfate concentrations for six food groups (dairy, meat, fish, fruit, vegetables, and grains).

ExpoKids graphs were generated for both 1994-2010 and 2011-2016 to compare aggregate exposure before and after the 2010 start of the US endosulfan phase-out. Figure 3 shows aggregate ADD and aggregate LADD by lifestage ExpoKids graphs for dietary intake of endosulfan sulfate prior to 2010 (left column) and after 2010 (right column). For both time periods, infants experienced the largest ADD and the ADD estimates declined as age increased. In addition, graphs from both time periods showed that all childhood lifestages showed similar LADDs and that the adult LADD was highest. Based on PDP 
Fig. 3 Endosulfan sulfate illustrative case example graphs: ADD and LADD by lifestage before the 2010 endosulfan US phase-out are shown in the left column and after the phase-out in the right column.

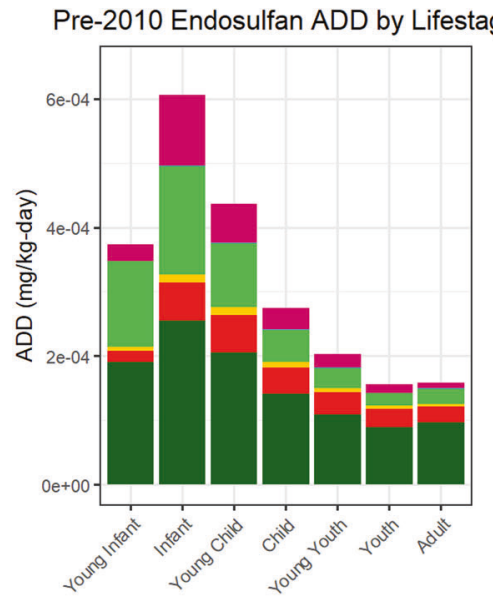

Post-2010 Endosulfan ADD by Lifestage
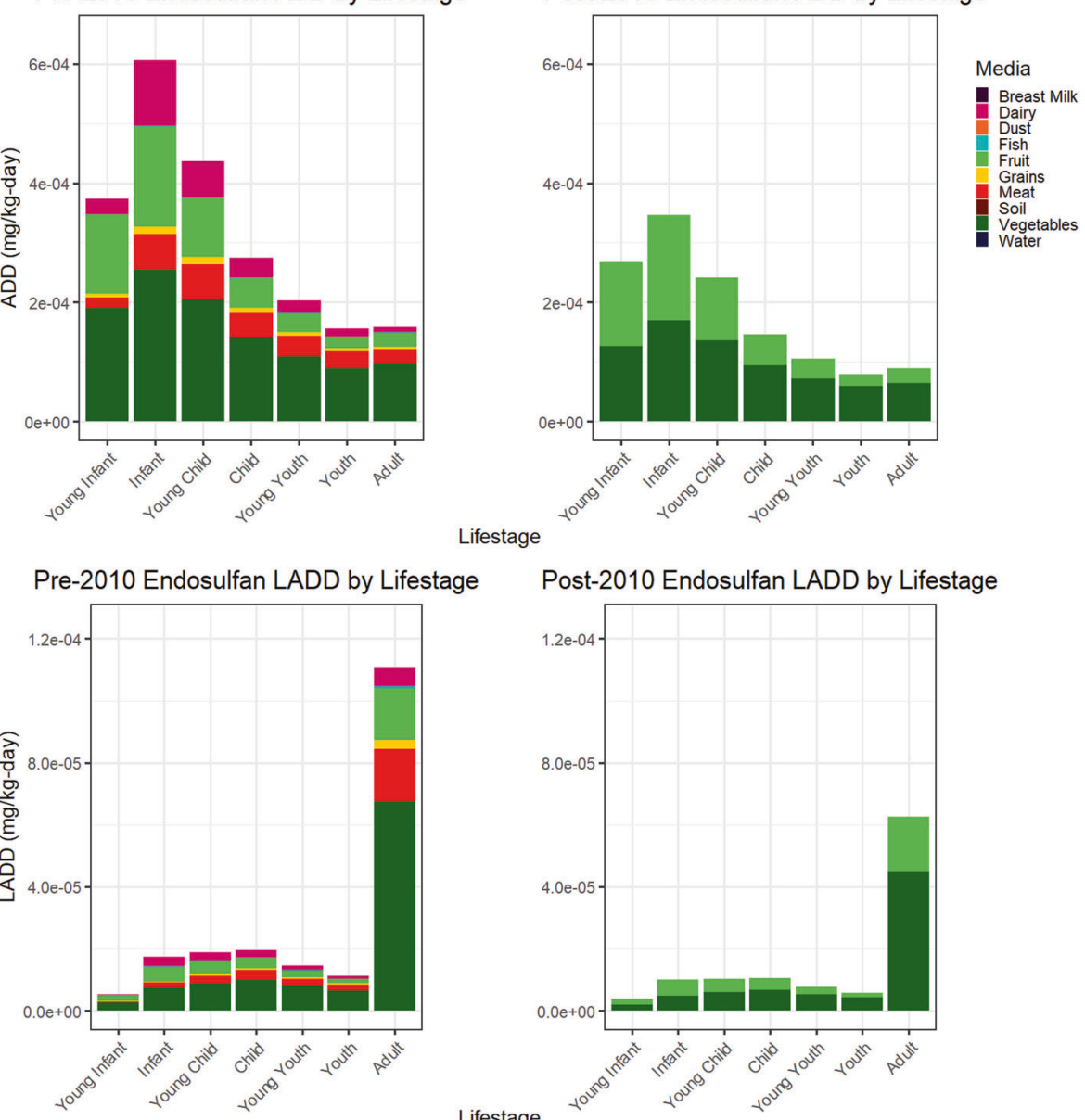

Lifestage

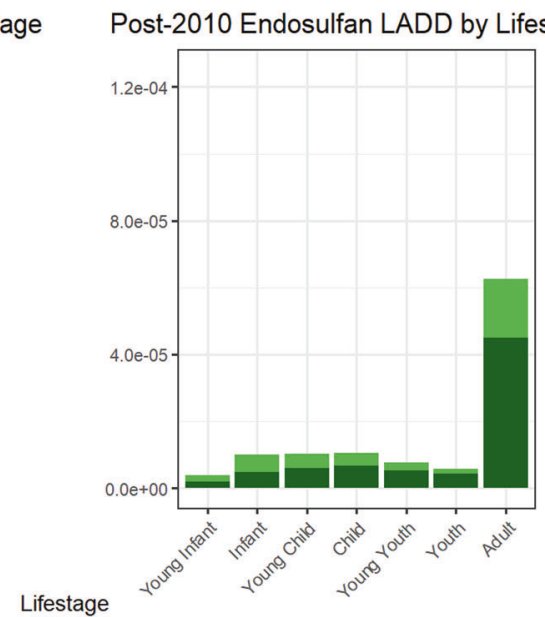

concentration data collected over these two time periods, endosulfan sulfate residues decreased to below analytical detection limits in all media except for fruits and vegetables for all lifestages after 2010. This would be expected since the crops whose last endosulfan usage dates occurred after 2010 were largely fruits and vegetables [36].

\section{Discussion}

ExpoKids integrates information for a single route of exposure (oral) from multiple media across lifestages to visually display lifetime estimated aggregate chemical exposures for childhood and adult lifestages. In exploring publicly available tools, ExpoKids is the only identified exposure assessment tool that depicts aggregate exposure to any chemical for multiple lifestages using this set of illustrative comparative graphs. However, EPA's exposure toolbox (ExpoBox; https://www.epa.gov/expobox) lists a variety of other tools that have also been developed for modeling aggregate exposure. Other proprietary tools may exist but are not publicly available. While these tools are powerful for estimating various exposures with a variety of inputs and specificity, each is built for a particular purpose that lacks the flexible data visualization of ExpoKids. For instance, SHEDS focuses on human activity patterns for chemical concentration and gathers exposure data from EPA field studies and published literature, IEUBK primarily estimates blood lead levels, and CEM was developed for consumer exposure scenarios [46-48]. These tools primarily provide calculated table outputs and lack the data visualization outputs that ExpoKids provides.

Since ExpoKids is coordinated with ExpoFIRST outputs, ExpoKids has the advantage of utilizing the recommended EFH lifestage-specific exposure factors. It can illustrate lifestage-specific aggregate exposure estimates for any chemical with media-specific exposure information. The ExpoKids v1.0 code (S-2) was developed in R, a publicly available, open-source software, for transparency. The user guide and code are available in the Supplementary Information (S-1 and S-2) and future updates to the R tool can be found on the EPA's ExpoKids webpage (www.epa. gov/expobox/expokids-data-visualization-tool-aggregate- 
exposure-lifestage-and-media). Although these case examples were estimated using ExpoFIRST v2.0, an updated ExpoFIRST v2.1 was released in June 2019. ExpoKids was developed with ExpoFIRST ADD calculations in mind, but runs separately. Therefore, as ExpoFIRST revisions reflect EFH updates, ExpoKids can continue to display ADDs calculated with the most recent exposure factors. ExpoKids also has the flexibility to visually display exposure estimates for multiple scenarios, as exemplified in the three illustrative case examples. The DEHP case example highlighted individual media contributions to aggregate exposure across developmental lifestages, revealing both specific behaviors and dietary patterns that could impact lifestage aggregate exposures. The manganese case example showed the lifestage- and medium-specific contributions of these exposures relative to established health benefit and toxicity levels. The endosulfan case example depicted decreased media concentrations and lifestage exposure rates following the US phase-out and ban of endosulfan. Yet these illustrative case examples addressed only a fraction of the range of questions that could be addressed using ExpoKids.

Scoping decisions simplified data acquisition and input for the illustrative case examples. For instance, ExpoKids combined the smallest age groups from ExpoFIRST into the young infant and infant lifestages, resulting in a loss of specific information for newborn ADD. Since sample size was not a selection criterion, some studies with small sample sizes $(n<30)$ were included. Moreover, per capita central tendency estimates did not capture population variability; actual aggregate exposure likely varied across different populations. For example, ADDs estimated for groups with specific characteristics (e.g., families living near a contaminated area) may be higher compared to the general public. The ADD estimation also assumed that the media concentrations remained constant over time for all lifestages. This fixed chemical concentration captured only a snapshot of the population's exposure at one point in time; to look at exposure as the population ages, temporal exposure concentration data would be needed. Similarly, the LADD was estimated separately for each lifestage and therefore, averaged the exposure for each lifestage over a lifetime. This limitation, inherent to the LADD equation, resulted in LADDs that did not account for ingestion during other lifestages. Therefore, total lifetime exposure may have been underestimated. Due to the limited availability of sex-specific data, differences in exposure by sex were not evaluated. Similarly, ExpoKids graphs did not represent comprehensive aggregate exposure from all routes and media for a given chemical due to a lack of chemical-specific data and the limited number of included media groups. Even though not all exposures were captured in ExpoKids, knowledge on the oral route of exposure was still gained.
ExpoKids also shared limitations with ExpoFIRST. For instance, point estimates were calculated rather than probabilistic distributions. ExpoFIRST does not use physiologically based pharmacokinetic (PBPK) models, so internal doses were not estimated. ExpoFIRST also does not evaluate the prenatal lifestage, although gestation may be a critical window of exposure; current data gaps render some exposure estimates during the gestational lifestage uncertain. In addition, the effectiveness of ExpoKids relies on the chemical concentration data that users input into ExpoFIRST and the exposure parameters selected. ExpoFIRST v2.0 did not have an option to evaluate infant formula consumption even though supplementing breastmilk with infant formula is commonly practiced in the USA; studies have indicated that $\sim 24 \%$ of 12-month-olds breastfeed [49]. As a result, ExpoFIRST v2.0 overestimated breastmilk consumption and did not represent formula-fed infants who would have increased water consumption. After the development of ExpoKids, EPA updated the EFH (Chapter 3; https://cfpub.epa.gov/ ncea/risk/recordisplay.cfm?deid $=343661$ ) to include data on drinking water intake among formula-fed infants to address this data gap. Therefore, ADD calculations made with these and future data updates may be used to calculate ADDs for visualization in ExpoKids.

\section{Challenges and opportunities}

ExpoKids contributes to an improved understanding of relative aggregate exposure by lifestage for various oral media. ExpoKids can facilitate the investigation of a broad range of questions beyond those covered by the illustrative case examples, e.g., evaluating whether multiple chemicals with a similar mode of action reach a level of concern when the chemicals individually may not. For environmental chemical decisions requiring region-specific chemical concentration information, ExpoKids can be used by scientists to assess the aggregate risk among different geographic areas. Specifically, it could be asked: Do aggregate exposures differ for different settings (e.g., urban vs. rural)? Do they correlate with non-environmental factors (sex, behavior, regulation, etc.)? ExpoFIRST and ExpoKids could be used to explore these and other risk assessment questions for available exposure data from limited media for the oral route of exposure.

Some limitations of ExpoKids reflected data constraints. More publicly available data for chemical-specific media concentrations are needed. At this time, chemical monitoring can vary by media; however, comprehensive contaminant data for all media sources are needed to improve aggregate exposure modeling. For many developmentally toxic chemicals, the most sensitive critical window of exposure is expected to occur during prenatal development. Unfortunately, most chemicals 
lack a PBPK model for gestation due to data gaps. Yet some chemicals, including those presented in these illustrative case examples, have prenatal and postnatal critical windows. Therefore, developing methods to incorporate gestational exposure is crucial. In addition, data collected from multiple compatible data sources or from meta-analyses would offer a more comprehensive aggregate exposure assessment and allow for customized analyses in the future, depending on the availability of user-defined data identifying specific factors (i.e., sex, age, etc.).

By combining the functionality of ExpoFIRST with the visual graphic capabilities of $\mathrm{R}$, ExpoKids facilitates comparisons of aggregate exposure by media both within and across lifestages. A broad range of ExpoKids users is envisioned. These include chemical risk assessors and managers running ExpoKids as a scoping tool to identify relevant exposure questions, as well as teachers using ExpoKids as an aggregate exposure teaching tool. Other potential users, such as pediatric health experts, may wish to use ExpoKids to explore differences in consumption patterns and exposure factors between and within children's lifestages. Consequently, potential users can focus on relative contributions from different media to estimate the impacts of possible mitigation technologies or policies. Future efforts include enhancing public availability of this tool by converting ExpoKids into a web-based R Shiny application. As more exposure data for environmental chemicals become available, the capabilities of ExpoKids may be expanded.

\section{Code availability}

The ExpoKids v1.0 user guide $(\mathrm{S}-1)$ and $\mathrm{R}$ code $(\mathrm{S}-2)$ are provided in the Supplementary Information.

Acknowledgements The authors would like to thank Brenda Foos for her suggestion of a children's health aggregate exposure project. We appreciate the thoughtful and productive discussions about the tool and the chemical illustrations with EPA's Office of Children's Health Protection, Office of Water, Office of Pollution Prevention and Toxics, Office of Pesticides, and Office of Research and Development scientists. We are also grateful to Zaida Figueroa, Rebecca Dzubow, and Lisa Melnyk, and to the anonymous reviewers for their thoughtful comments on the presubmission and submitted draft manuscripts. We gratefully acknowledge Maureen Johnson for developing the EPA ExpoKids website.

Funding This research was supported in part by an appointment to the Internship/Research Participation Program at the Office of Children's Health Protection, U.S. Environmental Protection Agency, administered by the Oak Ridge Institution for Science and Education (ORISE) through an interagency agreement between the US Department of Energy and EPA.

\section{Compliance with ethical standards}

Conflict of interest The authors declare that they have no conflicts of interest.
Publisher's note Springer Nature remains neutral with regard to jurisdictional claims in published maps and institutional affiliations.

Open Access This article is licensed under a Creative Commons Attribution 4.0 International License, which permits use, sharing, adaptation, distribution and reproduction in any medium or format, as long as you give appropriate credit to the original author(s) and the source, provide a link to the Creative Commons license, and indicate if changes were made. The images or other third party material in this article are included in the article's Creative Commons license, unless indicated otherwise in a credit line to the material. If material is not included in the article's Creative Commons license and your intended use is not permitted by statutory regulation or exceeds the permitted use, you will need to obtain permission directly from the copyright holder. To view a copy of this license, visit http://creativecommons. org/licenses/by/4.0/.

\section{References}

1. Guidance on Selecting Age Groups for Monitoring and Assessing Childhood Exposures to Environmental Contaminants. Washington, DC: US Environmental Protection Agency; 2005. Report No.: EPA/630/P-03/003F.

2. U.S. EPA. Exposure Factors Handbook 2011 Edition (Final Report). Washington, DC: U.S. Environmental Protection Agency; 2011. Report No.: EPA/600/R-09/052F.

3. Risk Assessment Forum. Guidelines for developmental toxicity risk assessment. Washington, DC: US Environmental Protection Agency; 1991. Report No.: EPA/600/FR-91/001.

4. Food Quality Protection Act. Sect. 405, 104-70; 1996. Available online at: https://www.govinfo.gov/content/pkg/PLAW-104publ170/ pdf/PLAW-104publ170.pdf.

5. U.S. EPA. General principles for performing aggregate exposure and risk assessments. Office of Pesticide Programs. Item \# 6043. 2001. Available online at: https://www.epa.gov/sites/production/ files/2015-07/documents/aggregate.pdf.

6. Frank R. Lautenberg Chemical Safety for the 21 st Century Act. Public Law 114-182. [H.R. 2576]; 2016. Available online at: https://www.congress.gov/114/plaws/publ182/PLAW114publ182.pdf.

7. Agency for Toxic Substances and Disease Registry (ATSDR). Framework for assessing health impacts of multiple chemicals and other stressors. Atlanta, GA: US Department of Health and Human Services, Public Health Service; 2018.

8. Danish Ministry of the Environment, Environmental Protection Agency. Guidance for risk assessment of chemicals in consumer articles and products. Survey of chemical substances in consumer products No. 125, 2014. The Danish Environmental Protection Agency; 2014. Available online at: https://www2.mst.dk/Udgiv/ publications/2014/03/978-87-93178-21-2.pdf.

9. Canada, Pest Management Regulatory Agency, Alternative Strategies and Regulatory Affairs Division. General principles for performing aggregate exposure and risk assessments; 2003.

10. Meek MEB, Boobis AR, Crofton KM, Heinemeyer G, Van Raaij M, Vickers C. Risk assessment of combined exposure to multiple chemicals: A WHO/IPCS framework. Regul Toxicol Pharmacol. 2011;60:S1-14.

11. A summary of general assessment factors for evaluating the quality of scientific and technical information. Washington, DC: US Environmental Protection Agency; 2003. Report No.: EPA 100/B-03/001.

12. U.S. EPA. Di(2-ethylhexyl)phthalate (DEHP; CASRN 117-81-7) Integrated Risk Information System (IRIS) Chemical Assessment Summary. National Center for Environmental Assessment. 
Washington, DC: U.S. Environmental Protection Agency; 1987. Available online at: https://cfpub.epa.gov/ncea/iris/iris_documents/ documents/subst/0014_summary.pdf.

13. US Consumer Product Safety Commission (CPSC). Chronic hazard advisory panel on phthalates and phthalate alternatives [Internet]. Bethesda, MD: US Consumer Product Safety Commission Directorate for Health Sciences; 2014. https://www.cpsc. gov/chap.

14. WHO. Guidelines for drinking-water quality. Fourth edition, incorporating the 1st addendum. Switzerland: World Health Organization (WHO); 2017. Available online at: https://apps.who. int/iris/bitstream/handle/10665/254637/9789241549950-eng.pdf; jsessionid=E22EDD7AB63FB597C988E92C54877B3F? sequence $=1$.

15. Minnesota Department of Health (MDH). Toxicological Summary for: Di-(2-ethylhexyl) phthalate. Saint Paul, MN: Minnesota Department of Health; 2015. p. 13. Report No.: 651-201-4899.

16. Subedi B, Sullivan KD, Dhungana B. Phthalate and non-phthalate plasticizers in indoor dust from childcare facilities, salons, and homes across the USA. Environ Pollut. 2017;230:701-8.

17. Lin Z, Zhang J, Cui H, Zhang L, Chen G. Determination of phthalate esters in soil by microemulsion electrokinetic chromatography coupled with accelerated solvent extraction. J Sep Sci. 2010;33:3717-25.

18. Loraine GA, Pettigrove ME. Seasonal variations in concentrations of pharmaceuticals and personal care products in drinking water and reclaimed wastewater in Southern California. Environ Sci Technol. 2006;40:687-95.

19. Hartle JC, Cohen RS, Sakamoto P, Barr DB, Carmichael SL. Chemical contaminants in raw and pasteurized human milk. J Hum Lact. 2018;34:340-9.

20. Schecter A, Lorber M, Guo Y, Wu Q, Yun SH, Kannan K, et al. Phthalate concentrations and dietary exposure from food purchased in New York State. Environ Health Perspect. 2013;121:473-94.

21. Institute of Medicine (US) Panel on Micronutrients. Dietary reference intakes for vitamin A, vitamin $\mathrm{K}$, arsenic, boron, chromium, copper, iodine, iron, manganese, molybdenum, nickel, silicon, vanadium, and zinc. Washington, DC: National Academies Press (US); 2001.

22. U.S. EPA. Manganese (CASRN 7439-96-5) Integrated Risk Information System (IRIS) Chemical Assessment Summary. National Center for Environmental Assessment. Washington, DC: U.S. Environmental Protection Agency, 1988. Available online at: https://cfpub.epa.gov/ncea/iris/iris_documents/documents/subst/ 0373_summary.pdf.

23. Grant D, Blazak WF, Brown GL. The reproductive toxicology of intravenously administered MnDPDP in the rat and rabbit. Acta Radiol. 1997;38:759-69.

24. Golub MS, Hogrefe CE, Germann SL, Tran TT, Beard JL, Crinella FM, et al. Neurobehavioral evaluation of rhesus monkey infants fed cow's milk formula, soy formula, or soy formula with added manganese. Neurotoxicol Teratol 2005;27:615-27.

25. Deskin R, Bursian SJ, Edens FW. Neurochemical alterations induced by manganese chloride in neonatal rats. Neurotoxicology 1981;2:65-73.

26. Chung SE, Cheong H-K, Ha E-H, Kim B-N, Ha M, Kim Y, et al. Maternal blood manganese and early neurodevelopment: the Mothers and Children's Environmental Health (MOCEH) Study. Environ Health Perspect 2015;123:717-22.

27. Claus Henn B, Ettinger AS, Schwartz J, Téllez-Rojo MM, Lamadrid-Figueroa H, Hernández-Avila $M$, et al. Early postnatal blood manganese levels and children's neurodevelopment. Epidemiol Camb Mass 2010;21:33-9.

28. Chan D. Development of health criteria for school site risk assessment pursuant to health and safety code section 901(g): child-specific reference doses (chRDs) for school site risk assessment, manganese and pentachlorophenol. Sacramento, CA: Office of Environmental Health Hazard Assessment; 2006. p. 70.

29. Health Canada. Guidelines for Canadian drinking water quality: guideline technical document - Manganese. Ottawa, Ontario: Health Canada; 2019. 114.

30. World Health Organization. Manganese in drinking-water: background document for development of WHO guidelines for drinking-water quality. WHO/SDE/WSH/03.04/104. Geneva, Switzerland: World Health Organization; 2004. Available online at: https://apps.who.int/iris/bitstream/handle/10665/75376/WHO_ SDE_WSH_03.04_104_eng.pdf? sequence $=1 \&$ isAllowed $=\mathrm{y}$.

31. Gulson B, Mizon K, Taylor A, Korsch M, Davis JM, Louie H, et al. Pathways of $\mathrm{Pb}$ and $\mathrm{Mn}$ observed in a 5-year longitudinal investigation in young children and environmental measures from an urban setting. Environ Pollut. 2014;191:38-49.

32. Klein LD, Breakey AA, Scelza B, Valeggia C, Jasienska G, Hinde $\mathrm{K}$. Concentrations of trace elements in human milk: comparisons among women in Argentina, Namibia, Poland, and the United States. PLoS ONE 2017;12:e0183367.

33. Lindsey, B.D. and Johnson, T.D. Data from Decadal Change in Groundwater Quality Web Site, 1988-2014, Version 2.0: U.S. Geological Survey data release. 2018. Available online at: https://doi.org/10.5066/F7N878ZS.

34. Total diet study: market baskets 2006 through 2013. College Park, MD: US Food and Drug Administration (FDA); 2017.

35. Brown MT, Foos B. Assessing children's exposures and risks to drinking water contaminants: a manganese case study. Hum Ecol Risk Assess 2009;15:923-47.

36. U.S. EPA. Endosulfan: Final Product Cancellation Order. Federal Register Notice. November 10, 2010. 75 FR 69065. Document \#: 2010-28138. pp.: 69065-69069. Washington, DC: U.S. Environmental Protection Agency, 2010. Available online at: https://www.federalregister.gov/documents/2010/11/10/201028138/endosulfan-final-product-cancellation-order.

37. WHO. Guidelines for drinking-water quality. 3rd edition. Volume 1 Recommendations. Geneva, Switzerland: World Health Organization. 2004. pp. 368-369. Available online at: https://www.who.int/water_sanitation_health/dwq/ GDWQ2004web.pdf.

38. Re-evaluation Note REV2011-01, Discontinuation of Endosulfan. Pest Management Regulatory Agency, Health Canada, Ottawa, Ontario, Canada. February 8, 2011. ISSN: 1925-0649 (PDF version). Available online at: https://www.canada.ca/content/dam/hcsc/migration/hc-sc/cps-spc/alt_formats/pdf/pubs/pest/decisions/ rev2011-01/rev2011-01-eng.pdf.

39. Janssen MPM. Endosulfan. A closer look at the arguments against a worldwide phase out. Bilthoven, Netherlands: National Institute for Public Health and the Environment; 2011, p. 92. Report No.: 601356002/2011.

40. Cory-Slechta DA, Thiruchelvam M, Richfield EK, Barlow BK, Brooks AI. Developmental pesticide exposures and the Parkinson's disease phenotype. Birt Defects Res A Clin Mol Teratol. 2005;73:136-9.

41. Moreno Frías M, Jiménez Torres M, Garrido Frenich A, Martínez Vidal JL, Olea-Serrano F, Olea N. Determination of organochlorine compounds in human biological samples by GCMS/MS. Biomed Chromatogr Bmc. 2004;18:102-11.

42. Jimenez Torres M, Campoy Folgoso C, Cañabate Reche F, Rivas Velasco A, Cerrillo Garcia I, Mariscal Arcas M, et al. Organochlorine pesticides in serum and adipose tissue of pregnant women in Southern Spain giving birth by cesarean section. Sci Total Environ. 2006;372:32-8.

43. Wilson WW, Shapiro LP, Bradner JM, Caudle WM. Developmental exposure to the organochlorine insecticide endosulfan damages the nigrostriatal dopamine system in male offspring. 
NeuroToxicology 2014;44:279-87.

44. USDA. Pesticide data program. United States Department of Agriculture; 2017. Available online at: https://www.ams.usda. gov/sites/default/files/media/2017PDPDatabase.ZIP.

45. Ghadiri H. Degradation of endosulfan in a clay soil from cotton farms of western Queensland. J Environ Manag. 2001;62: $155-69$.

46. Zartarian VG, Ozkaynak H, Burke JM, Zufall MJ, Rigas ML, Furtaw EJ. A modeling framework for estimating children's residential exposure and dose to chlorpyrifos via dermal residue contact and nondietary ingestion. Environ Health Perspect. 2000;108:505-14.
47. Lee RC, Fricke JR, Wright WE, Haerer W. Development of a probabilistic blood lead prediction model. Environ Geochem Health. 1995;17:169-81.

48. Office of Pollution Prevention and Toxics (OPPT). Approaches to estimate consumer exposure under TSCA. Washington, DC: US Environmental Protection Agency; 2019. Available online at: https://www.epa.gov/tsca-screening-tools/approaches-estimateconsumer-exposure-under-tsca.

49. Grummer-Strawn LM, Scanlon KS, Fein SB. Infant feeding and feeding transitions during the first year of life. Pediatrics 2008;122 (Suppl 2):S36-42. 